\title{
The psychometric properties of the Montreal Cognitive Assessment (MoCA): A comprehensive investigation
}

Giovanni Sala ${ }^{1}$, Hiroki Inagaki ${ }^{2}$, Yoshiko Ishioka ${ }^{3}$, Yukie Masui ${ }^{2}$, Takeshi Nakagawa ${ }^{4}$, Tatsuro Ishizaki ${ }^{2}$, Yasumichi Arai ${ }^{5}$, Kazunori Ikebe ${ }^{6}$, Kei Kamide ${ }^{7}$, and Yasuyuki Gondo ${ }^{8}$

${ }^{1}$ Institute for Comprehensive Medical Science, Fujita Health University, Toyoake, Japan.

${ }^{2}$ Tokyo Metropolitan Institute of Gerontology, Tokyo, Japan.

${ }^{3}$ Graduate School of Science and Technology, Keio University, Yokohama, Japan.

${ }^{4}$ National Center for Geriatrics and Gerontology, Aichi, Japan.

${ }^{5}$ Center for Supercentenarian Medical Research, Keio University, Tokyo, Japan.

${ }^{6}$ Graduate School of Dentistry, Osaka University, Osaka, Japan.

${ }^{7}$ Graduate School of Medicine, Osaka University, Osaka, Japan.

${ }^{8}$ Graduate School of Human Sciences, University of Osaka, Osaka, Japan.

Funding. This work was supported by the Japan Society for the Promotion of Science [17F17313, 17H02633]; and the Ministry of Education, Culture, Sports, Science, and Technology of Japan [21330152, 26310104].

\section{Author's Note}

Corresponding author: Giovanni Sala, Institute for Comprehensive Medical Science (ICMS), 1-98 Dengakugakubo Kutsukake-cho, Toyoake, Fujita Health University 4701192, Japan. E-mail: sala.giovanni@fujita-hu.ac.jp.

Word count: 2,426

Tables: 3

\section{Acknowledgements}

We gratefully thank Fred Oswald and Yves Rosseel for their assistance with the analyses. We also thank Fernand Gobet for providing comments on an earlier draft. 


\section{Abstract}

\section{Background}

The Montreal Cognitive Assessment (MoCA) is a common tool for the assessment of global cognition in older adults. Despite its popularity among clinicians and researchers, the test's psychometric properties are still uncertain.

\section{Objective}

To examine fundamental psychometrics properties of the MoCA that have not been established so far (i.e., factorial structure, general factor saturation, and measurement invariance).

\section{Design}

Cohort study.

\section{Setting}

Population-based invitation-type survey in city and rural areas in Hyogo prefectures and Tokyo Metropolitan, Japan.

\section{Subjects}

Individuals $(N=2,408)$ aged 69 to 91 clustered in three cohorts (69-71-year-olds, 79-81year-olds, and 89-91-year-olds).

\section{Methods}

Exploratory Factor Analysis and Confirmatory Factor Analysis.

\section{Results}

The MoCA shows an overall stable hierarchical factorial structure and a satisfactory general factor saturation. Also, measurement invariance occurs across participants with different age, educational level, economic status, and gender.

\section{Conclusion}

This comprehensive investigation upholds the idea that the MoCA is a psychometrically valid tool for the assessment of global cognition in older adults.

\section{Key points}

- The Montreal Cognitive Assessment (MoCA) is a common test for evaluating global cognition in older adults. 


\section{PSYCHOMETRICS OF THE MOCA}

- The MoCA has never undergone a thorough analysis to test its psychometric properties.

- The study evaluates the test's dimensionality, factorial structure, general factor saturation, and invariance.

- The results show that the test's items perform well in all the analyses.

- The MoCA is thus a reliable tool for assessing global cognition in older adults.

Keywords: cognition; factor analysis; measurement invariance; MoCA; older adults. 


\section{PSYCHOMETRICS OF THE MOCA}

\section{Introduction}

The Montreal Cognitive Assessment (hereafter MoCA) [1] is one of the most common tests for measuring global cognition and detect potential global cognitive impairment in older adults. Administering the MoCA is quick (approximately ten minutes), and the test can be used with both older adults and old-older adults (e.g., > 80-year-old). The test is thus a useful and convenient instrument for the study of cognitive function (and dysfunction). For these reasons, the MoCA is currently employed by researchers and clinicians all over the world, as testified by the numerous citations of Nasreddine and colleagues' article (more than 10,000 citations in Google Scholar, as of December 2019).

The MoCA has been designed to measure a construct of interest, that is, the subject's global cognition. Consequently, the MoCA total score is supposed to be a measure of this construct. Nonetheless, some fundamental psychometric properties of the MoCA's items are still unclear. Specifically, their factorial structure, reliability, and measurement invariance have not been satisfactorily investigated so far. Without establishing these properties, any inferences based on the MoCA's scores remain doubtful.

When analyzing the factorial structure of a test's items, three cases are possible. First, only one latent general factor is present (i.e., unidimensionality). In this case, the test does measure just one construct of interest with a certain degree of reliability. Second, more than one latent factor is estimated (i.e., multidimensionality), but no general factor is present. In this case, the total test score is not particularly meaningful because it does not refer to any general construct. Finally, the following case is possible: the factorial structure of the test is multidimensional but, at the same time, all the test items correlate with each other, which suggests the presence of a general factor. In this case, the total test score is indeed a measure of the putative general factor. However, the reliability of the test's total score cannot be estimated with an index such as Cronbach's $\alpha$. In fact, like any other total factor saturation index, $\alpha$ is not trustworthy when the assumption of unidimensionality is not met [2]. Rather, an index of general factor saturation - that is, the proportion of the total 


\section{PSYCHOMETRICS OF THE MOCA}

score variance of a test accounted for by a single common factor [3] - is necessary to evaluate the reliability of the test correctly (e.g., McDonald's $\omega$ and Revelle's $\beta$ ) [4].

Whether the MoCA score reflects only one general factor (unidimensionality) or more factors (with or without a general factor) is still a matter of debate. In some studies, unidimensionality has simply been postulated $[1,5]$. Other studies have provided some evidence that the MoCA's items are substantially unidimensional [6,7]. However, these studies implement Generalized Partial Credit Modelling [8] that requires summing clusters of the MoCA's items into seven sub-scores (i.e., the clusters indicated by Nasreddine and colleagues). Although viable, this approach postulates, rather than tests, a specific structure in the data. Such a priori assumptions about the MoCA's data structure have also been employed with factor analysis $[9,10]$. In these cases, the MoCA's items have been found to be multidimensional with no general factor, but the number of factors is unclear. Finally, other researchers have adopted a more exploratory approach and highlighted the tendency of the MoCA's items to converge towards a multidimensional structure with a general factor [11]. However, no index of general factor saturation is provided.

Finally, no thorough analysis of measurement invariance has been performed on the MoCA so far. This gap in the literature is particularly concerning. Measurement invariance is a crucial property for any test because it concerns whether the test's total scores have the same meaning under a set of different conditions (e.g., between genders and across participants of different age). Without establishing measurement invariance, it is impossible to make any meaningful comparison across groups. Therefore, this state of affairs makes the interpretation of MoCA scores problematic.

There are several hierarchically organized levels of measurement invariance [12]. The least restrictive one is configural invariance, which denotes whether a test's items show the same factorial structure across conditions. Weak invariance (also referred to as metric invariance) assumes configural invariance and requires the same factor loadings across groups and thus establishes that the constructs measured by the test's total scores are manifested in the same way across groups. Simply put, this condition tells us that responses 


\section{PSYCHOMETRICS OF THE MOCA}

on the items refer to the latent constructs with the same metric across groups. Strong invariance (also referred to as scalar invariance) assumes weak invariance and requires the same intercepts across groups. Finally, the most restrictive level is strict invariance, which requires both strong invariance and equal items' variances across groups. Strict invariance, therefore, provides information about how precisely the construct of interest is measured.

This study implements a systematic analytical strategy to address the above issues. First, we establish whether the items of the MoCA subtend either a unidimensional or multidimensional (with or without a general factor) structure. Second, we use exploratory factor analysis (EFA) and confirmatory factor analysis (CFA) to determine the exact factorial structure of the MoCA. Third, we provide several reliability indexes for both general factor saturation and total factor saturation. Finally, we test for measurement invariance in four fundamental demographic variables - age, educational level, economic status, and gender - that may constitute confounding elements in the assessment of global cognition.

\section{Methods}

\section{Participants}

The study included a total of 2,408 Japanese older adults. The participants were organized in three cohorts accordingly to their age range (69-71, 79-81, and 89-91). The data were retrieved from the SONIC survey and referred to baseline assessment. All the details regarding the SONIC survey can be retrieved from [13].

\section{Variables}

MoCA. We used the data of the Japanese translation of the MoCA. For more details, see [14]. As in Freitas and colleagues' study [11], the 32 dichotomous (0/1) items of the test were used in the analyses.

Demographics variables. We examined gender (male, female), age cohort (see above), education, and self-reported economic status of the participants. Education included three levels indicating the highest educational degree achieved by the participant 


\section{PSYCHOMETRICS OF THE MOCA}

(primary/middle school, high school, and university/college education). The economic status of the participants included three levels as well (no financial leeway, some financial leeway, good financial leeway).

\section{Analytical approach}

We employed a systematic strategy. First, we calculated the percentage of correct responses in each item of the MoCA. Second, we ran several unidimensionality tests. Third, we ran an exploratory factor analysis (EFA) to investigate the factorial structure of the MoCA in a randomly selected subsample [2]. Fourth, the results of the EFA were tested with confirmatory factor analysis (CFA) that included all the observations not used in the EFA model. Finally, we tested measurement invariance of the MoCA score between genders, age cohorts, educational level, and self-reported economic status.

\section{Results}

\section{Descriptive statistics}

The descriptive statistics are summarized in Table 1.

Table 1

MoCA total scores sorted by age cohorts, educational levels, economic status, and gender

\begin{tabular}{|c|c|c|c|c|}
\hline Age/Cohort & 70-year-old & 80-year-old & 90-year-old & Total \\
\hline$N$ & 970 & 943 & 495 & 2,408 \\
\hline Mean (SD) & $24.03(3.66)$ & $22.28(4.29)$ & $20.24(5.15)$ & $22.56(4.48)$ \\
\hline Education & Primary/Middle & High School & College/University & Total \\
\hline$N$ & 824 & 889 & 695 & 2,408 \\
\hline Mean $(S D)$ & $20.36(4.61)$ & $23.53(3.94)$ & $23.94(3.95)$ & $22.56(4.48)$ \\
\hline Gender & Males & Females & - & Total \\
\hline$N$ & 1,138 & 1,270 & - & 2,408 \\
\hline Mean $(S D)$ & $22.64(4.26)$ & $22.50(4.66)$ & - & $22.56(4.48)$ \\
\hline Economic Status & No Leeway & Some Leeway & Good Leeway & Total \\
\hline$N$ & 488 & 1328 & 592 & 2,408 \\
\hline Mean (SD) & $21.86(4.48)$ & $22.53(4.52)$ & $23.22(4.28)$ & $22.56(4.48)$ \\
\hline
\end{tabular}




\section{PSYCHOMETRICS OF THE MOCA}

\section{Data preparation}

We examined the mean correct response rate on each of the 32 items of the MoCA. To avoid estimation problems related to ceiling effect (e.g., inflated item complexity in EFA), we excluded those items whose mean correct response was above $95 \%(n=5)$. All the following analyses were thus performed with the remaining 27 dichotomous items of the MoCA. In addition, in order to control for any differences introduced by this technical choice, we replicated the analyses including all the 32 items. Only negligible differences were found (see the Supplemental materials).

\section{Dimensionality tests}

We first ran a unidimensionality check on a Rasch model $[15,16]$. The analyses were performed with the $\operatorname{ltm} \mathrm{R}$ package [17,18]. This analysis showed evidence of multidimensionality $(p<.010)$. We then ran a parallel analysis [19] to establish the number

of first-order factors with the psych $\mathrm{R}$ package [20]. The tetrachoric correlation matrix and the weighted least squares (WLS) estimator were used. The parallel analysis estimated eight factors. The inspection of eigenvalues suggested the presence of a general factor (first eigenvalue $=6.40$, second eigenvalue $=1.67$ ).

\section{Exploratory Factor Analysis}

The previous analyses established that our data showed a multi-factorial structure. First, following standard guidelines [21], we randomly selected half of the participants $(N=$ 1,204; calibration sample), while the other half of the sample was used in the CFA analysis (see below). We then ran a hierarchical EFA with seven first-order factors and assuming a general factor, as indicated by the parallel analysis. We performed the analyses with the psych $\mathrm{R}$ package. All the items loaded onto the general factor $(g)$. The seven sub-factors approximatively corresponded to the seven subsets indicated by Nasreddine and colleagues. Overall, the items thus exhibited a stable multi-factorial structure. Table 2 summarizes the results of the EFA. 


\section{PSYCHOMETRICS OF THE MOCA}

Table 2

Results of the EFA model

\begin{tabular}{|c|c|c|c|c|c|c|c|c|c|c|}
\hline MoCA Item & $g$ & $F 1$ & $F 2$ & $F 3$ & $F 4$ & $F 5$ & F6 & $F 7$ & $h 2$ & $u 2$ \\
\hline Q1.TRAIL MAKING & 0.54 & 0.09 & -0.03 & 0.10 & 0.20 & 0.16 & 0.31 & 0.03 & 0.47 & 0.53 \\
\hline Q2.CUBE & 0.41 & 0.05 & 0.12 & -0.10 & 0.24 & 0.00 & $\mathbf{0 . 3 5}$ & 0.02 & 0.40 & 0.60 \\
\hline Q3.CLOCK HAND & 0.44 & -0.08 & 0.03 & 0.08 & -0.03 & 0.03 & 0.45 & 0.10 & 0.41 & 0.59 \\
\hline Q3.CLOCK SHAPE & 0.49 & 0.04 & -0.01 & 0.07 & -0.01 & 0.03 & 0.49 & 0.04 & 0.48 & 0.52 \\
\hline Q4.NAMING CAMEL & 0.47 & -0.02 & -0.01 & 0.19 & -0.03 & 0.50 & 0.02 & 0.04 & 0.50 & 0.50 \\
\hline Q4.NAMING LION & 0.38 & -0.02 & 0.00 & -0.05 & 0.06 & 0.66 & -0.03 & 0.04 & 0.60 & 0.40 \\
\hline Q4.NAMING RHINO & 0.52 & 0.15 & 0.13 & -0.02 & -0.11 & 0.48 & 0.17 & -0.02 & 0.58 & 0.42 \\
\hline Q6.DIGIT SPAN BACKWARD & 0.34 & 0.08 & 0.14 & -0.03 & 0.41 & 0.01 & 0.05 & 0.02 & 0.32 & 0.68 \\
\hline Q6.DIGIT SPAN FORWARD & 0.36 & 0.01 & 0.04 & -0.01 & 0.39 & 0.11 & 0.10 & 0.04 & 0.32 & 0.68 \\
\hline Q7.ATTENTION & 0.43 & 0.13 & 0.05 & 0.17 & 0.24 & 0.04 & 0.07 & 0.03 & 0.30 & 0.70 \\
\hline Q8.SUBTRACTION 86 & 0.41 & -0.02 & 0.40 & 0.12 & 0.10 & -0.05 & 0.12 & -0.09 & 0.39 & 0.61 \\
\hline Q8.SUBTRACTION 79 & 0.42 & 0.01 & 0.62 & -0.04 & -0.02 & 0.05 & 0.05 & -0.04 & 0.57 & 0.43 \\
\hline Q8.SUBTRACTION 72 & 0.46 & -0.01 & 0.74 & 0.01 & 0.01 & -0.02 & -0.06 & 0.05 & 0.76 & 0.24 \\
\hline Q8.SUBTRACTION 65 & 0.47 & 0.02 & 0.54 & 0.06 & 0.00 & 0.05 & 0.03 & 0.02 & 0.51 & 0.49 \\
\hline Q9.WORD REPETITION 1 & 0.31 & 0.01 & 0.04 & 0.04 & 0.41 & -0.10 & 0.02 & 0.19 & 0.33 & 0.67 \\
\hline Q9.WORD REPETITION 2 & 0.39 & -0.08 & 0.12 & 0.15 & 0.30 & 0.15 & -0.07 & 0.09 & 0.32 & 0.68 \\
\hline Q10.VERBAL FLUENCY & 0.39 & 0.11 & 0.04 & 0.00 & 0.20 & 0.25 & 0.11 & -0.02 & 0.28 & 0.72 \\
\hline Q11.ABSTRACTION 1 & 0.37 & 0.03 & 0.01 & -0.03 & 0.03 & -0.01 & 0.03 & 0.64 & 0.54 & 0.46 \\
\hline Q11.ABSTRACTION 2 & 0.40 & 0.07 & -0.01 & 0.10 & -0.03 & 0.05 & 0.05 & 0.44 & 0.37 & 0.63 \\
\hline Q12.RECALL 1 & $\mathbf{0 . 3 2}$ & 0.55 & 0.05 & 0.08 & -0.16 & -0.04 & -0.01 & 0.08 & 0.47 & 0.53 \\
\hline Q12.RECALL 2 & 0.39 & 0.61 & 0.03 & -0.04 & 0.00 & 0.10 & -0.05 & 0.09 & 0.53 & 0.47 \\
\hline Q12.RECALL 3 & 0.45 & $\mathbf{0 . 5 8}$ & -0.04 & 0.12 & 0.18 & 0.05 & -0.04 & -0.02 & 0.61 & 0.39 \\
\hline Q12.RECALL 4 & 0.41 & 0.68 & 0.04 & -0.03 & 0.01 & -0.07 & 0.12 & -0.03 & 0.64 & 0.36 \\
\hline Q12.RECALL 5 & 0.40 & 0.67 & -0.03 & 0.05 & 0.00 & 0.03 & -0.02 & 0.02 & 0.63 & 0.37 \\
\hline Q13.ORIENTATION YEAR & 0.63 & -0.07 & 0.07 & 0.72 & 0.00 & 0.02 & 0.02 & 0.06 & 0.90 & 0.10 \\
\hline Q13.ORIENTATION DAY & 0.60 & 0.20 & 0.00 & 0.65 & 0.01 & -0.03 & 0.02 & -0.03 & 0.88 & 0.12 \\
\hline Q13.ORIENTATION PLACE & 0.43 & -0.04 & -0.06 & 0.38 & 0.08 & 0.16 & 0.08 & 0.00 & 0.35 & 0.65 \\
\hline
\end{tabular}

Note. Item $=$ the item of the MoCA; $g=$ general factor; $F 1=$ factor $1 ; F 2=$ factor $2 ; F 3=$ factor $3 ; F 4=$ factor $4 ; F 5=$ factor $5 ; F 6=$ factor $6 ; F 7=$ factor $7 ; h^{2}=$ communality of the item; $u^{2}=$ uniqueness of the item. 


\section{PSYCHOMETRICS OF THE MOCA}

\section{Confirmatory Factor Analysis}

We built a hierarchical CFA model following the results of the EFA. We used the other half of the sample. This model included seven first-order factors loading onto one second-order factor. Since the indicators were dichotomous, we used the WLSMV estimator. We performed these analyses with the lavaan and semTools R packages [22,23].

We calculated the statistical power for not-close fit hypothesis testing [24]. Assuming the null RMSEA $=.050$, alternative $\mathrm{RMSEA}=.010$, the statistical power was more than adequate $(>99 \%)$ for global fit testing and rejection of false models $(5 \%$ significance threshold). The CFA model exhibited good fit, $\chi^{2}{ }_{(317)}=453.776, p<.001$, $\mathrm{RMSEA}=.019$, and $\mathrm{SRMR}=.063, \mathrm{CFI}=.970$, and $\mathrm{NCI}=.972[25]$. The model estimated by the EFA analysis was thus confirmed by the CFA model in an independent sample.

\section{Reliability indexes}

We calculated McDonald's omega $(\omega)$ coefficients and Revelle's beta $(\beta)$ coefficient to measure the general factor saturation of the MoCA. The percentage of the reliable (i.e., not due to random error) variance - the ratio between omega hierarchical $\left(\omega_{h}\right)$ and omega total $\left(\omega_{t}\right)$ multiplied by 100 [3] - in the MoCA total scores accounted for by the general factor $g$ was satisfactory $\left(74 \% ; \omega_{h}=.68\right.$ and $\left.\omega_{t}=.92\right)$. Revelle's beta, which represents the minimum split-half reliability, was adequate too $(\beta=.78)$. Total factor saturation indexes were similar to $\omega_{t}$ (Cronbach's $\alpha=.89$ and Guttman's $\lambda_{6}=.91$ ).

\section{Measurement invariance}

Finally, we tested whether the MoCA's items exhibited configural, weak, and strict invariance across the age groups, education level, gender, and economic status of the participants (strong invariance cannot be tested with dichotomous indicators). We performed these analyses on the whole sample $(N=2,408)$ with the semTools $\mathrm{R}$ package.

The analyses were run according to the guidelines provided by Chen and colleagues, Rudnev and colleagues, and $\mathrm{Wu}$ and Estabrook [26-28]. First, configural invariance was tested by imposing the model's threshold to be equal across groups. Second, we tested two types of weak invariance by imposing equality constraints to (a) the factor 


\section{PSYCHOMETRICS OF THE MOCA}

loading between first-order factors and the observed variables (i.e., the items of the test; Weak $-1^{\text {st }}$ ) and then (b) all the factor loadings (Weak - Full). Finally, strict invariance was first tested by applying equality constraints to the first-order factors' variances (the variances of the indicators were fixed to 1 in the configural model) [28].

Measurement invariance was tested by inspecting the difference $(\Delta)$ in fit indexes in increasingly constrained models (e.g., configural vs. weak). The thresholds for model rejection were: $\triangle \mathrm{RMSEA}>.015, \Delta \mathrm{SRMR}>.030, \Delta \mathrm{CFI}<-.010$, and $\Delta \mathrm{NCI}<-.020$ $[29,30]$. Measurement invariance was confirmed for all the four demographic variables. None of the fit indexes reached its threshold for model rejection (maximum $\triangle \mathrm{RMSEA}=$ $+.001 ; \Delta \mathrm{SRMR}=+.005 ; \Delta \mathrm{CFI}=-.003 ; \Delta \mathrm{NCI}=$-.006). Moreover, the chi-squared different test, whose Type I error rate is notoriously high, barely reached statistical significance in only one case $(p=.044)$. Table 3 summarizes the results of this analysis.

Table 3

Summary of the Measurement Invariance Analysis

\begin{tabular}{|c|c|c|c|c|c|c|c|}
\hline & $\chi^{2}$ & $d f$ & p-value & $R M S E A$ & SRMR & CFI & $N C I$ \\
\hline \multicolumn{8}{|l|}{ Age } \\
\hline Configural & 1171.289 & 930 & - & .018 & .079 & .972 & 0.951 \\
\hline Weak-1st & 1213.124 & 970 & .207 & .018 & .082 & .972 & 0.951 \\
\hline Weak-Full & 1252.537 & 982 & .044 & .019 & .087 & .969 & 0.945 \\
\hline Strict & 1277.572 & 996 & .085 & .019 & .087 & .967 & 0.943 \\
\hline \multicolumn{8}{|l|}{ Education } \\
\hline Configural & 1172.227 & 930 & - & .018 & .077 & .970 & 0.951 \\
\hline Weak-1st & 1214.340 & 970 & .229 & .018 & .081 & .970 & 0.951 \\
\hline Weak-Full & 1208.505 & 982 & .545 & .017 & .082 & .972 & 0.954 \\
\hline Strict & 1234.239 & 996 & .075 & .017 & .083 & .970 & 0.952 \\
\hline \multicolumn{8}{|l|}{ Gender } \\
\hline Configural & 793.382 & 620 & - & .015 & .058 & .983 & 0.965 \\
\hline Weak-1st & 782.599 & 640 & .923 & .014 & .059 & .986 & 0.971 \\
\hline Weak-Full & 799.263 & 646 & .093 & .014 & .061 & .985 & 0.969 \\
\hline Strict & 800.430 & 653 & .754 & .014 & .061 & .985 & 0.970 \\
\hline
\end{tabular}


PSYCHOMETRICS OF THE MOCA

\begin{tabular}{llrrrrrr}
\hline Wealth & \multicolumn{7}{l}{} \\
\hline Configural & 1135.111 & 930 & - & .017 & .071 & .978 & 0.958 \\
\hline Weak-1st & 1153.828 & 970 & .505 & .015 & .073 & .981 & 0.963 \\
\hline Weak-Full & 1150.744 & 982 & .515 & .015 & .074 & .982 & 0.966 \\
\hline Strict & 1157.154 & 996 & .636 & .014 & .075 & .983 & 0.967 \\
\hline
\end{tabular}

Note $\cdot \chi^{2}=$ Scaled Chi Squared; $D f=$ Degrees of freedom of the model; $p$-value $=$ significance of the scaled Chi-Squared difference test; $R M S E A=$ Root Mean Square Error of Approximation (scaled); $S R M R=$ Standardized Root Mean Square Residual; $C F I=$ Comparative Fit Index (scaled); NCI = Non-Centrality Index (scaled).

\section{Discussion}

The aim of this paper was to evaluate the psychometric properties of the MoCA's items in a large sample of Japanese older adults. Specifically, we have investigated (a) the dimensionality of the MoCA's items; (b) their factorial structure; (c) their total factor saturation and general factor saturation; and (d) whether measurement invariance occurs across a set of fundamental demographic variables such as age, education, economic status, and gender. The analytical strategy implemented includes a set of exploratory methods (parallel analysis and EFA) and confirmatory methods (CFA and measurement invariance analysis). This choice has allowed us to cross-validate the factorial structure of the test's items in two independent samples without applying any a priori data structure (e.g., summing the items according to Nasreddine et al.’s categorization [1]).

The results show that the MoCA is, overall, a valid tool for assessing global cognition in older adults. First, the presence of a general factor - along with seven subfactors - indicates that the test's total score is indeed a measure of global cognition. This factorial structure is verified in both the EFA and CFA models. Second, the reliability of the MoCA's items is adequate in terms of both total factor saturation $\left(\alpha=.89\right.$ and $\left.\lambda_{6}=.91\right)$ and, most notably, general factor saturation $\left(\omega_{h} / \omega_{t}=.74\right.$ and $\left.\beta=.78\right)$. Third, measurement invariance occurs is confirmed from the least restrictive model (configural invariance) to the most restrictive model (strict invariance) in all the demographic variables examined. This finding is of particular interest because no previous investigation has ever included a 


\section{PSYCHOMETRICS OF THE MOCA}

comprehensive measurement invariance analysis of the MoCA. (It is worth noting that Luo et al. [7] have tested measurement invariance analysis between subjects with and without formal education in a Chinese sample and found that most of the items are invariant.)

Furthermore, the present study may offer some insights regarding the inconsistent outcomes provided by the literature so far. As seen, measurement invariance analysis and general factor saturation have substantially remained untested. By contrast, the research on the MoCA's dimensionality and factorial structure has been somewhat more abundant but has produced mixed results. Our investigation corroborates the assumption of a general factor made by several previous studies [1,5-7]. The only difference is that these studies report that the general factor is also the only latent dimension in the data (unidimensionality). The difference with our results, which suggest multidimensionality in the MoCA's items, simply stems from a methodological choice. In fact, we have not imposed any a priori constraint to the data (e.g., summing sets of items) that would conceal the true hierarchical factorial structure of the test's items.

\section{Conclusions}

The present study reports a comprehensive psychometric analysis of the MoCA. The test shows a stable hierarchical factorial structure, satisfactory general factor saturation, and measurement invariance in key demographic variables. The MoCA is thus a reliable tool for assessing global cognition in older adults. 


\section{References}

1. Nasreddine ZS, Phillips NA, Bédirian V, Charbonneau S, Whitehead V, Collin I, et al. The Montreal Cognitive Assessment, MoCA: A brief screening tool for mild cognitive impairment. J Am Geriatr Soc. 2005;53: 695-699. doi:10.1111/j.15325415.2005.53221.x

2. Zinbarg RE, Revelle W, Yovel I, Li W. Cronbach's $\alpha$, Revelle's $\beta$ and McDonald's $\omega$ h: Their relations with each other and two alternative conceptualizations of reliability. Psychometrika. 2005;70: 123-133. doi:10.1007/s11336-003-0974-7

3. Reise SP, Bonifay WE, Haviland MG. Scoring and modeling psychological measures in the presence of multidimensionality. J Pers Assess. 2013;95: 129-140. doi:10.1080/00223891.2012.725437

4. Zinbarg RE, Yovel I, Revelle W, McDonald RP. Estimating generalizability to a latent variable common to all of a scale's indicators: A comparison of estimators for wh. Appl Psychol Meas. 2006;30: 121-144. doi:10.1177/0146621605278814

5. Delgado C, Araneda A, Behrens MI. Validation of the Spanish-language version of the Montreal Cognitive Assessment test in adults older than 60 years. Neurol (English Ed. 2019;34: 376-385. doi:10.1016/J.NRLENG.2018.12.008

6. Freitas S, Prieto G, Simões MR, Santana I. Scaling cognitive domains of the Montreal Cognitive Assessment: An analysis using the Partial Credit Model. Arch Clin Neuropsychol. 2015;30: 435-447. doi:10.1093/arclin/acv027

7. Luo H, Andersson B, Tang JYM, Wong GHY. Applying Item Response Theory analysis to the Montreal Cognitive Assessment in a low-education older population. Assessment. 2019. doi:10.1177/1073191118821733

8. Thomas ML. The value of item response theory in clinical assessment: A review. Assessment. 2011;18: 291-307. doi:10.1177/1073191110374797

9. Duro D, Simões MR, Ponciano E, Santana I. Validation studies of the Portuguese experimental version of the Montreal Cognitive Assessment (MoCA): confirmatory factor analysis. J Neurol. 2010;257: 728-734. doi:10.1007/s00415-009-5399-5

10. Coen RF, Robertson DA, Kenny RA, King-Kallimanis BL. Strengths and limitations of the MoCA for assessing cognitive functioning: Findings from a large representative sample of Irish older adults. J Geriatr Psychiatry Neurol. 2016;29: 1824. doi:10.1177/0891988715598236

11. Freitas S, Simões MR, Marôco J, Alves L, Santana I. Construct validity of the Montreal Cognitive Assessment (MoCA). J Int Neuropsychol Soc. 2012;18: 242250. doi:10.1017/S1355617711001573 


\section{PSYCHOMETRICS OF THE MOCA}

12. Millsap RE, Yun-Tein J. Assessing factorial invariance in ordered-categorical measures. Multivariate Behav Res. 2004;39: 479-515.

doi:10.1207/S15327906MBR3903 4

13. Gondo Y, Masui Y, Kamide K, Ikebe K, Arai Y, Ishizaki T. SONIC Study: A longitudinal cohort study of the older people as part of a centenarian study. In: Nancy P, editor. Encyclopedia of Geropsychology. Singapore: Springer; 2016. doi:10.1007/978-981-287-080-3

14. Suzuki H, Kawai H, Hirano H, Yoshida H, Ihara K, Kim H, et al. One-year change in the Japanese version of the Montreal Cognitive Assessment performance and related predictors in community-dwelling older adults. J Am Geriatr Soc. 2015;63: 1874-1879. doi:10.1111/jgs.13595

15. Drasgow F, Lissak RI. Modified parallel analysis: A procedure for examining the latent dimensionality of dichotomously scored item responses. J Appl Psychol. 1983;68: 363-373. doi:10.1037/0021-9010.68.3.363

16. Rasch G. Probabilistic models for some intelligence and achievement tests. Copenhagen Danish Inst Educ Res. 1960. doi:10.1016/S0019-9958(61)80061-2

17. R Core Team. R: A language and environment for statistical computing. http://www.R-project.org/. In: R Foundation for Statistical Computing, Vienna, Austria. 2017.

18. Rizopoulos D. 1tm: An R Package for Latent Variable Modeling. J Stat Softw. 2006.

19. Hayton JC, Allen DG, Scarpello V. Factor retention decisions in exploratory factor analysis: A tutorial on parallel analysis. Organ Res Methods. 2004;7: 191-205. doi: $10.1177 / 1094428104263675$

20. Revelle MW. psych: Procedures for personality and psychological research (R package). Evanston, Illinois: Northwestern University; 2017.

21. Kyriazos TA. Applied Psychometrics: The 3-Faced Construct Validation Method, a Routine for Evaluating a Factor Structure. Psychology. 2018;9: 2044-2072. doi:10.4236/psych.2018.98117

22. Rosseel Y. lavaan : An R Package for Structural Equation Modeling. J Stat Softw. 2015;48: 1-36. doi:10.18637/jss.v048.i02

23. Terrence J, Pornprasertmanit S, Alexander S, Rosseel Y. semTools: Useful tools for structural equation modeling. R package version 0.5-1. 2018. Available:

https://cran.r-project.org/package $=$ semTools

24. Kline RB. Principles and practice of structural equation modeling (2nd ed.). Methodology in the social sciences. 2005. doi:10.1038/156278a0

25. Hu LT, Bentler PM. Cutoff criteria for fit indexes in covariance structure analysis: 


\section{PSYCHOMETRICS OF THE MOCA}

Conventional criteria versus new alternatives. Struct Equ Model. 1999;6: 1-55. doi:10.1080/10705519909540118

26. Chen FF, Sousa KH, West SG. Testing measurement invariance of second-order factor models. Struct Equ Model. 2005;12: 471-492.

doi:10.1207/s15328007sem1203_7

27. Rudnev M, Lytkina E, Davidov E, Schmidt P, Zick A. Testing measurement invariance for a second-order factor: A cross-national test of the alienation scale. Methods, Data, Anal. 2018;12: 47-76. doi:10.12758/mda.2017.11

28. Wu H, Estabrook R. Identification of Confirmatory Factor Analysis models of different levels of invariance for ordered categorical outcomes. Psychometrika. 2016;81: 1014-1045. doi:10.1007/s11336-016-9506-0

29. Chen FF. Sensitivity of goodness of fit indexes to lack of measurement invariance. Struct Equ Model. 2007;13: 464-504. doi:10.1080/10705510701301834

30. Cheung GW, Rensvold RB. Evaluating goodness-of-fit indexes for testing measurement invariance. Struct Equ Model. 2002;9: 233-255.

doi:10.1207/S15328007SEM0902_5 